EPJ Web of Conferences 41, 05024 (2013)

DOI: $10.1051 /$ epjconf/20134105024

(C) Owned by the authors, published by EDP Sciences, 2013

\title{
Controlling Quantum Interferences in IR Vibrational Excitations in Metal Carbonyls
}

\author{
S. Ashihara ${ }^{1}$, K. Enomoto ${ }^{1}$, J. Tayama $^{1}$ \\ ${ }^{1}$ Department of Applied Physics, Tokyo University of Agriculture and Technology, \\ 2-24-16, Nakacho, Koganei, Tokyo 184-8588, Japan
}

\begin{abstract}
Coherent controls over vibrational excitations in metal di-carbonyls were demonstrated by using phase-shaped mid-infrared pulses. The phase of the inter-state coherence between two different normal-modes was controlled by changing the phase step of the stepwise spectral phase profile. The quantum interference between multiple vibrational excitation paths in resonant two-step excitations was manipulated by the similar spectral phase control. The results experimentally confirmed that coherent control over multiple vibrational degrees of freedom works properly in condensed phases. There remains the possibility for increasing excitation efficiency by introducing group-delays and by optimizing polarization state of the excitation pulse.
\end{abstract}

\section{Motivation and Objectives}

Coherent control is a technique that manipulates interference of wave functions by adjusting their amplitudes and phases. Recent developments in the pulse shaping technique in MIR [1] may open a way toward coherent control over molecular motions/reactions at the electronic ground states. Such control may serve for novel information processing and molecular reaction controls, including bond breakage, structural isomerization, hydrogen bond rearrangement, proton transfer, etc. [2-4]. So far the MIR pulse shaping was applied to the ladder climbing of a single vibrational mode [5].

The broadband nature of the ultrashort MIR pulses, however, should also be able to excite multiple vibrational modes in a correlated manner to control nuclear motion in multi-dimensional coordinates and to create highly-excited vibrational states. The ability to handle multiple vibrational modes should increase the variety and efficiency of controllable reactions. For such coherent control applications, control over quantum interference as well as efficient ladder climbing is essential. In this paper, we show that the quantum interferences in IR vibrational excitations were successfully controlled for liquid-phase molecules by use of MIR phase-shaping.

\section{MIR Pulse Shaping and Transient Absorption Spectroscopy}

MIR pulses of $100-$ fs duration and $5-\mu \mathrm{J}$ energy were generated by the OPA/DFG system. Major portion of the pulse energy was delivered to the pulse shaper, consisting of diffraction gratings, concave mirrors and the germanium AOM, developed by Zanni-group [1]. The shaped pulse was characterized by the electric-field cross correlation in the spectral interferometry with a reference

This is an Open Access article distributed under the terms of the Creative Commons Attribution License 2.0, which permits unrestricted use, distribution, and reproduction in any medium, provided the original work is properly cited. 
pulse, which had been characterized by an interferometric auto-correlation. With this setup, we achieved higher-order dispersion compensation and precise phase shaping [6]. The shaped pulse and the unshaped pulse with fractional energy were used as pump and probe pulses, respectively. The pump and probe pulses had linear polarizations, parallel to each other. The spectra of probe pulses transmitted through a sample were acquired by a monochromator and a $\mathrm{HgCdTe}$ detector array.

\section{IR Coherent Control Experiments on Metal Carbonyls}

\subsection{Controlling the phase of the interstate coherence between two normal modes}

Our samples were metal di-carbonyls $\operatorname{Ir}(\mathrm{CO})_{2} \mathrm{C}_{5} \mathrm{H}_{7} \mathrm{O}_{2}$ (IDC) and $\mathrm{Rh}(\mathrm{CO})_{2} \mathrm{C}_{5} \mathrm{H}_{7} \mathrm{O}_{2}$ (RDC), which have been model compounds in 2D-IR spectroscopy: vibrational couplings and population/coherence transfer in metal carbonyls have been studied [7-9]. The vibrational energy-level diagram for two anharmonically coupled symmetric (S) and anti-symmetric (A) $\mathrm{CO}$ stretch vibrations is shown in Fig.1(a). Here $\left|m_{\mathrm{S}} n_{\mathrm{A}}\right\rangle$ denotes the eigenstate with quantum numbers $\mathrm{m}$ and $\mathrm{n}$ for $\mathrm{S}$ and $\mathrm{A}$ modes, respectively, and $\phi_{i}$ denotes the phase of the MIR frequency component relevant for each transition. The center frequency of the pump with $200-\mathrm{cm}^{-1}$ bandwidth was tuned so that the pump spectrum covers six transitions (shown as arrows).

Figure 1(b) shows the transient absorption spectrum at 1-ps time delay for IDC in $n$-hexane, pumped by nearly transform-limited pulses. The linear absorption for IDC (a dashed line) reveals the fundamental transition frequencies of 1999 and $2074 \mathrm{~cm}^{-1}$ for A and S modes, respectively. There observed the bleaching signal at the fundamental transition frequency for each mode and the corresponding excited-state absorption. The inset shows transient signals at 2065 and $2074 \mathrm{~cm}^{-1}$. On top of the excited-state absorption/bleaching contributions, the oscillations with a beat frequency of the two states $\left|0_{\mathrm{S}} 1_{\mathrm{A}}\right\rangle$ and $\left|1_{\mathrm{S}} 0_{\mathrm{A}}\right\rangle$ ( $\sim 40$ fs period) were observed, indicating the coherent superposition of the two states.

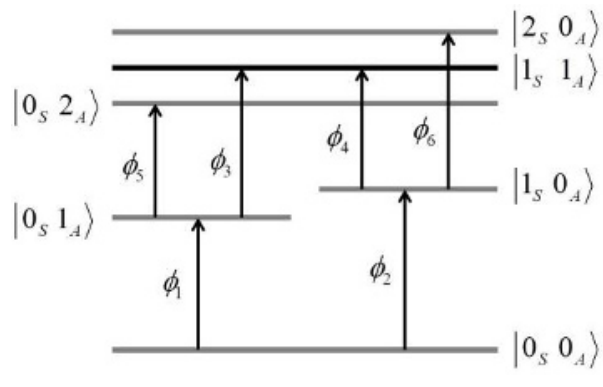

(a)

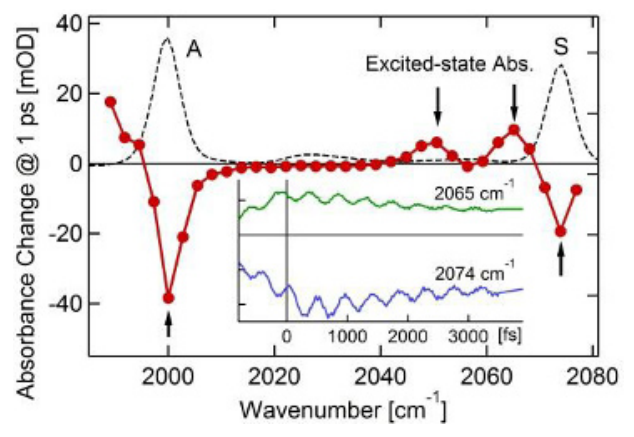

(b)

Fig. 1. (a) Vibrational energy-level diagram for IDC and RDC. (b) The transient absorption spectrum measured at time-delay of 1 ps pumped with nearly transform-limited pulses: the inset shows the transient signals at 2065 and $2074 \mathrm{~cm}^{-1}$.

Now the spectral phase of the pump was shaped in a stepwise profile to control $\phi_{2}-\phi_{1}$. Figure 2(a) shows the beat signals extracted from the transients probed at $2074 \mathrm{~cm}^{-1}$. As can be seen, the phase of the beat signal changed according to the relative phase $\phi_{2}-\phi_{1}$. Superposition of energetically close but non-degenerate two modes creates $2 \mathrm{D}$ vibrational trajectory, which evolves with time (as linear, circular, or elliptic). Therefore the present control corresponds to selecting the initial trajectory upon excitation. This is also interpreted as control over local-mode amplitudes at the moment of excitation. 


\subsection{Controlling multiple-paths interference in two-step excitations}

We used similar phase-shaping scheme to control the excitation efficiency into $\left|1_{\mathrm{S}} 1_{\mathrm{A}}\right\rangle$ state for $\mathrm{RDC}$ in $n$-hexane. It is expected that there exist two of the resonant two-step excitation paths toward $\left|1_{\mathrm{S}} 1 \mathrm{~A}\right\rangle$ state: the one via $\left|0_{\mathrm{S}} 1_{\mathrm{A}}\right\rangle$ and the other via $\left|1_{\mathrm{S}} 0_{\mathrm{A}}\right\rangle$. Then the efficiency of excitation into the $\left|1_{\mathrm{S}} 1_{\mathrm{A}}\right\rangle$ state, because of the quantum interference, should depend on the phase difference $\Delta \phi=$ $\left(\phi_{1}+\phi_{3}\right)-\left(\phi_{2}+\phi_{4}\right)$. Figure 2(b) shows the absorbance change at $\left|1_{\mathrm{S}} 1_{\mathrm{A}}\right\rangle \rightarrow\left|1_{\mathrm{S}} 2_{\mathrm{A}}\right\rangle$ transition frequency $\left(1975 \mathrm{~cm}^{-1}\right)$ measured while the phase $\phi_{4}$ was varied. As is clearly seen, the absorbance change (proportional to the $\left|1_{\mathrm{S}} 1_{\mathrm{A}}\right\rangle$ population) varied sinusoidally with a period of $2 \pi$. This indicates that the quantum interference between multiple excitation paths into $\left|1_{\mathrm{S}} 1_{\mathrm{A}}\right\rangle$ state was controlled by the spectral phase. Additional set of experiments revealed that there are more than two excitation paths, some of which are activated by coherence transfers between $\mathrm{S}$ and A excitations.

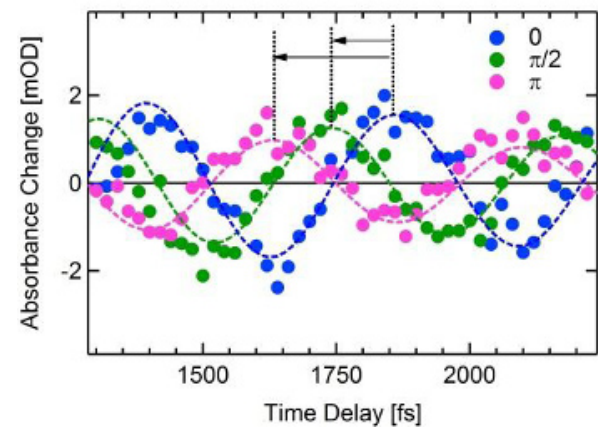

(a)

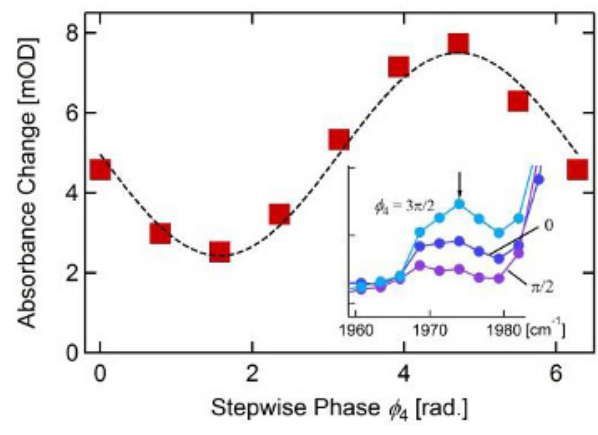

(b)

Fig. 2. (a) The beat signal at $2074 \mathrm{~cm}^{-1}$ probe frequency for IDC when the pump had the stepwise spectral phase of $0,0.5 \pi$, and $1.0 \pi$, respectively. (b) The phase $\phi_{4}$ dependence of the absorbance change reflecting the $\left|1_{\mathrm{S}} 1_{\mathrm{A}}\right\rangle$ population for RDC.

\section{Summary}

The inter-state coherence between two vibrational modes and the quantum interference between multiple vibrational excitation paths in metal di-carbonyls were controlled by the phase-shaped MIR pulses. The results confirmed that IR coherent control over multiple vibrational degrees of freedom works properly in condensed phases. Future works include the enhancement of the two-step excitation efficiency, by introducing group-delays and by optimizing the polarization states.

\section{References}

1. S. -H. Shim, D.B. Strasfeld, E.C. Fulmer, and M.T. Zanni, Opt. Exp. 14, 13120 (2006).

2. Y. Fujimura, L. Gonzalez, K. Hoki, J. Manz, Y. Ohtsuki, Chem. Phys. Lett. 306, 1 (1999).

3. M. Artamonov, T.-S. Ho, H. Rabitz, Chem. Phys. 328, 147 (2006).

4. C. Gollub M. Kowalewski, S. Thallmair and R. Vivie-Riedle, Phys. Chem. Chem. Phys. 12, 15780 (2010).

5. D.B. Strasfeld, S. -H. Shim, and M.T. Zanni, Phys. Rev. Lett. 99, 038102 (2007).

6. S. Ashihara, Y. Hirasawa, K. Enomoto, Conference on Lasers and Electro-Optics - European Quantum Electronics Conference, CF5.4 (2011).

7. M. Khalil, N. Demirdoven, A. Tokmakoffet al., J. Chem. Phys. 121, 362 (2004).

8. F. Ding, E.C. Fulmer, M.T. Zannia., J. Chem. Phys. 123, 094502 (2005).

9. C.R. Baiz, K.J. Kubarych, E. Geva, J. Phys. Chem. B 115, 5322 (2011). 